\title{
Critical Pedagogy as a Response to Datafication
}

\author{
Annette N. Markham'
}

\begin{abstract}
Critical pedagogy is a vital part of building data literacy. It moves beyond the level of data critique to social action in response to datafication. This article contends that academics can do more to teach those in the public sphere as well as classroom to become critical interpretive researchers of their own lived experience, an action/participatory research framework that identifies critical thinking as the purpose of research and improved digital or data literacy as the outcome of research. This article suggests three strategic modes through which the strengths of critical approaches and qualitative epistemologies can be blended to serve as pedagogical tools for understanding and critically analyzing data, datafication, and other aspects of the digital era.
\end{abstract}

\section{Keywords}

critical pedagogy, data literacy, datafication, participatory research, qualitative epistemologies

\section{Introduction}

The pace of digital technology development has outstripped the pace of reflection for more than 20 years now. Although we experience many benefits, it is well established by now that most regular citizens only superficially understand how digital services and platforms work. This includes impoverished knowledge about how our data is gathered and used (by, e.g., companies who design platforms using incomprehensible algorithms) or how computational processes impact what we see (e.g., advertising), what we don't see (e.g., numerous companies buying or selling our data), and how decisions are made on our behalf (e.g., news feeds or music recommendations). Critical awareness and curiosity are required to push back against forces that yield invasive target marketing, uninteresting Netflix recommendations, biased search results, overly narrow newsfeeds, or other problems encountered on a daily basis. Exacerbating this problem, most smart city and smart home technologies are embedded, difficult to observe or critique. As machine learning (AI) systems grow more nuanced, automated decision-making floods more of everyday life. Critical pedagogy, combined with a strong qualitative orientation, can challenge quantification, datafication, and computational logics. Rather than trying to defy the trend to use the term data, which at this point looks to be (read: is) a losing battle, I argue that qualitative researchers can play a proactive role by intervening in how everyday citizens interpret their own definitions of what counts as data, as these definitions get negotiated through their own lived experience within digitally saturated environments. This can be achieved by helping people become qualitative researchers of their own experience, to identify how identities, events, and cultural formations are quantified, and more importantly, to interrogate the ways their personal behaviors and activities and relationships - in the form of information - might be harvested, stored, and eventually commoditized for sale in the corporate or governmental marketplace.

Taking on this role requires that we academics reach outside the isolation and protection of the typical academic production cycle to teach as many people as possible to use our well-honed methodological tools and criti-cal (theory) approaches for thinking about their lives in this digital data epoch. After all, who better than well-trained social researchers to teach citizens to be researchers of their own lived experience? As we interact on formal and informal levels with audiences beyond the academy, we have the opportunity to help others analyze the way data flow in the situations they live in, how algorithmic logics intervene in citizen actions, how actions and responses in everyday interactions are constrained by the grammars of platform infrastructures, and how

\footnotetext{
'Aarhus University, Denmark
}

\section{Corresponding Author:}

Annette N. Markham, Professor, Department of Information Studies and Digital Design, Aarhus University, Aarhus, Denmark. Email: amarkham@gmail.com 
social media conversations and networked information function rhetorically to frame basic definitions of the self, relationships, politics, and institutional systems and dynamics.

To exemplify a critical pedagogy of the digitally oppressed, I describe an ongoing research project I started in 2012 to train youth (approximately 20-30 years old) to conduct selfreflexive ethnographic analysis of their own social media experiences. Although this experiment started as a technique for me as a researcher to obtain more fine-grained material about everyday lived experience in what we can call digitally saturated social contexts, it became immediately apparent that I was engaged in critical pedagogy (described in a talk by Markham \& Dougherty, 2014). Although the term originates with Paolo Freire's (1968) work in critical Pedagogy of the Oppressed, the concept itself indicates an interest in using methods of good teaching and learning (pedagogy) to raise critical consciousness about something. In the 1960s and 1970s, feminists used consciousness raising circles to build awareness of patriarchal patterns of society that reinforce gender roles and work against the interests of women. Italian social theorist Antonio Gramsci (1937/1971) also highlighted the importance of workers teaching themselves to be conscious of the conditions of structural oppression that hide beneath the surface of everyday institutional practices, a condition he labeled hegemony, or control through consent. Scandinavian Participatory Design can be considered another form of critical pedagogy, arising in the early 1970s in response to the perceived negative impacts of technology in the workplace. One underlying premise of the collective Nordic response over many years is that active participation is necessary for people to have a real influence on their working conditions (see Bødker, 2003). In this 2012-2018 study of social media and youth experience, participatory design merges with critical pedagogy, resulting in student-built layered reflexive ethnographic accounts that critically analyze their own and others' social media use.

Following a brief sketch of this ongoing research project, I offer some tips for a critical data pedagogy. These emerge from practice, so they should be read as contributions to broader ongoing conversations rather than a comprehensive list of what is possible.

\section{The Case: Teaching Others to Be Autoethnographers of Their Own Digital Lives}

Within a symbolic interactionist framework, lived experience is an ongoing negotiation of meaning, always within interactions. In the case of social media, interactions between people are mediated by various levels and types of technical and technological interfaces. Beginning with this premise, my project incorporates techniques from ethnography, autoethnography, and phenomenology, and is inspired by experiments and exercises that loosely follow the logic of Garfinkel's
(1967) ethnomethodological breaching experiments. This combination of methods unearths tacit norms, assumptions, practices, and feelings about an area of life that has been discursively overregulated by overly anxious and overly hopeful public sphere rhetoric since the early 1990s.

The design takes seriously and literally Freire's (1968) idea that respectful dialogue connects the personal experience to broader knowledge spheres. The educator, in this respect, knows more (i.e., I know more than the participants about the impact of social media, about ethnographic methods, about techniques of self-exploration through autoethnography), but includes the participant in the design of all the elements of the project. Thus, participants decide which technologies to study, how to track and document their use, and which genre and medium to formulate their reflections in. They decide how far to dive into the self or include others in their analysis. They decide what tools they'll use to document, describe, and share their experiences. Finally, they test different tools associated with inductive and emergent approaches (cf. Hesse-Biber \& Leavy, 2010) to find an approach that resonates with their own habitual and skilled ways of knowing.

Each set of participants met regularly for 10 to 12 sessions. The setup of each session was experimental and based in the foundations of what we generally mean by emergent or inductive approaches. More specifically, I used the vocabulary of remix, which coincides with the time period when I was reconceptualizing possible vocabularies for research methods that would allow people across disciplines to talk more effectively about what they were actually doing without getting stuck in disciplinary terminology (Markham, 2013).

Remix, as a way of describing both the process and product of inquiry, is enacted and accomplished through the activities of playing, borrowing, interrogating, moving, and generating. Participants were encouraged to use these terms when thinking about how they might track, log, and intensively analyze their everyday technology use. Such a playful approach yielded many methods, each providing a "way in" for accomplishing intensive, generative, and self-reflexive fieldwork.

Although flexible and varying with each cohort of participants, the basic process has always (during the past 6 years) included the following elements:

1. Tracking: Participants closely attended to every aspect of their digital media use for $48 \mathrm{hr}$, using different forms of tracking and logging techniques and tools.

2. Disconnecting: Participants would take a "media fast" for $24 \mathrm{hr}$ to experience disconnectivity. During this time, participants kept audio/video/text diaries to reflect on what it means and how it feels to be disconnected.

3. Reflecting: After each stage, participants wrote or recorded reflections in the form of brain dumps, drew situational maps building on the basic premises and techniques of Adele Clarke's (2003) situational analysis, and interviewed themselves and one other person. 
4. Interpreting: Following this intensive "generating" phase, they played around with different possible answers to questions like, "So what?" or "What does it mean?" or "Who cares?"

5. Repeating: After developing their initial interpretations, some participants would return to the field to repeat their observations, breaching experiments, recording, mapping, and reflecting, to develop richer ethnographic accounts. Depending on their level of interest at this point, they might also begin to include others in their study. Finally, some of them produced more refined multimedia narratives.

In years, I've collected well over 1,200 narrative accounts in multimedia format. As a whole, they are highly reflexive and show clear signs of consciousness raising. Many participants used a critical lens to analyze their social media use, which for a short time made them feel negative about themselves. Some believed they should stop using social media altogether. Most of this self-negativity wore off, as the complexity of the lived experience and the normative, commercial, and ideological discourses surrounding the Internet came into sharper relief. But the critical lens that accompanied these shifts in sense-making appears to remain as a tool to think about other situa-tions beyond their own personal interactions with and through digital and networked media. By the end of each experiment, which included many debriefing sessions and discussions about the inherent good/bad sides of media use, they could articulate a significantly more nuanced understanding of the continual struggle to find balance (Tiidenberg et al., 2017).

This has become a strong model for building critical data literacy. Iteratively developed and honed for 6 years, it has become a standard part of my own curriculum and has been taken up elsewhere with similarly strong outcomes. Although this project has generated a lot of valuable data about lived experience, this is an ancillary benefit. Rather, the point of the research is to do the research, and the findings are not for me, as the facilitator, but for the users who become researchers them-selves. What they do with these findings is up to them. In other words, once this experiment ends, my job is done. In this, I am functioning as a pedagogue more than an empirical researcher. The research outcome for me is not to provide answers but to raise questions and cause a chain reaction whereby participants raise their own questions and ask their parents, siblings, friends, and colleagues to also raise questions.

\section{Tips for Critical Pedagogy as Tool for Data Literacy:}

\section{Teach More Critical Theory, Without the Baggage Terminology of Critical Theory}

Critical theory can be an offputting subject for students less troubled than I am about data privacy or for those ensconced in neoliberal mind-sets of constant consumption and consumerism. Broaching the topic gradually and through examples and experiential experiments helps students reach for a critical mind-set, rather than accept one I'm trying to forcefeed them. I learned a trick from years of teaching organizational communication to students who were resistant to critical approaches: Build cognitive dissonance about a situation until the students insist on a critical perspective. To give a brief example, unrelated to digital technology, I often read aloud a shortened version of Shirley Jackson's short story The Lottery to students as a way of getting them to shift from descriptive "thick description" to "critical interrogation." As the story goes, the townspeople gather once a year in very ritualistic fashion for a lottery. The mystery of the story is that the reader doesn't have any idea what the lottery is for until the very end, and so at first, it seems like a straightforward, if quirky, example of the richness of everyday life. Taking a cultural perspective on organizing, students are instructed to consider these as fieldnotes from an ethnographer, and try to think about "what is going on here?" from a nonjudgmental perspective. After the story, I ask for comments and students inevitably address the wrongness of the situation.

The dialogue would go something like this:

\section{"That's horrible!"}

"I hear you," I reply, and I try to look sympathetic to soften my response, "But you know that's not really an analytical assessment. We're supposed to be ethnographers here. Maybe focus on some of the elements that could make this an organizational 'culture.' What sort of material objects or behaviors seem to point to a strong or cohesive culture?"

After some thought, another student says, "Well, there is the old saying that everyone seems to repeat, "lottery in June, corn be coming soon."

"Good,” I reply, "Anything else?"

Another student says, "They carry out the old box. It's tattered, but they treat it like it's a precious relic, so obviously it has significance in the culture."

I reply, "So does the box tell us anything about their cultural values?"

Another student erupts, "What do you mean, does the box tell us anything, Dr. Markham! What about them stoning someone to death? That would tell us something about their values!"

I reply, “Now, we agreed we wouldn't judge, but simply observe. Does anyone else have a comment?"

"But how can we ignore that the lottery 'winner' is killed by their neighbors and family?!" 
I repeat my stance, asking, "Well, if we're just observing a cultural practice, who are we to evaluate or critique?"

After awhile, I stop trying to play devil's advocate. I say, "I get the feeling that some of you are not satisfied with being value-neutral or simply descriptive. Maybe we should change our rules?"

If I inject this reading at the right point in the semester, it shocks bachelor students into not only accepting but insisting on the importance of a critical theory perspective in responding to a situation gone wrong, which could not be analyzed from a value-neutral perspective. ${ }^{1}$

The trick is not in the story but in letting students approach critical theory through watching or having an experience rather than reading about an abstract theoretical framework. There are many techniques for accomplishing this, but for me, it requires letting go of the baggage terms that go along with such approaches, such as interpellation, hegemony, and systematically distorted communication. Initially, I don't even use softer terms like disenfranchisement, disempowerment, and marginalization. Only later, once our everyday explanations fail to articulate an adequate accounting, can we (they) take up discipline-strengthened vocabularies and concepts to further develop the analytical accounts or build the frameworks or strategies for social action.

Even as I minimize the terminology associated with critical theory, I treat everything as a concern that requires a critical theory approach (more Foucault than Marx). This means every situation contains power imbalance and a struggle over meaning that privileges certain people and perspectives over others. Therefore, nothing is a neutral object to simply observe and describe. Likewise, there is no such thing as a value-neutral research project, which means that one's effort to address it ethically must take a stance in addition to a perspective. Combining standpoint and futureoriented approaches invites students to consider where structures come from, and how their argument has, or could have, impact on some future outcome.

This critical theory stance forms the foundation for anything we might call literacy, once we move beyond its classic definition of knowing how to read and write. Literacy-particularly digital literacy-has been mischaracterized in recent years to indicate competence in using digital tools, but a critical digital or data literacy requires far, more than simply being able to surf the Internet, use various platforms, carry out basic searches, and produce content.

A critical theory stance begins with the idea that there's something wrong and works to investigate the who, what, where, when, and how of this wrongness. Applied deliberately and consistently to everyday lived experiences, this stance is a strong response to datafication, as it questions everything from the perspective of "who benefits and who loses?"- a question in sharp contrast to the more typical open-ended qualitative question, "What is going on here?" The latter does not preclude a critical conclusion, but prompts a descriptive answer rather than a critical investigation. The first question is much more open for critically investigating the ideologically laden commercial, political, and populist discourses.

Young people are constantly criticized for being overly obsessed with consumption and consumerism. Simultaneously, they face limited job prospects and shrink-ing economies, forcing them to find a way of thriving in part-time and nomadic labor markets without a safety net. This precarity is not aided by thrusting critical theory upon them but by encouraging them to be deeply reflexive of their own experience, to question how larger structures come into being and end up dominating them in ways that may not be fair. I don't mean to say we should soft peddle critical the-ory - well, actually, that's exactly what I'm arguing. There's a strong need for critical theory yet a strong resistance to it from many quarters. Soft peddling it - by allowing it to emerge through the dialectical tensions within experiential and everyday inquiry (or qualitative research projects) - is one way to move these concepts into front burner territory while minimize their feelings of alienation or belittlement.

\section{Acknowledge (Big) Data as Relevant, Important, and Meaningful}

Within this era of datafication and data-oriented models for research, it is difficult to reject or avoid using the concept of data. It is the dominant term in use, particularly among funding agencies, from whom academics increasingly receive support for basic research. By now, the term data (as well as big data) has been thoroughly criticized (Boellstorff, 2013; boyd \& Crawford, 2012; Brock, 2015; Gitelman, 2013; Markham, 2013, 2017). Still, it is not going away any time soon, as a contemporary description of the stuff of social, political, technological, and material life.

Although tempting, it is also not enough to use terms like small data or thick data as a counterpoint to "big data," as Brock notes (2015). The strength of qualitative inquiry has never been about data in the first place, but about analysis, interpretation, and representation. These iterative and emer-gent processes of inquiry get short shrift in trends that privilege the up-front collection or production of information or mate-rial. Shifting the conversation away from data as thing or object-including its basic characteristics but also its collec-tion, storage and protection - and toward data as ideology (Markham, 2017) can highlight how data are - and might be otherwise - conceptualized more broadly. This requires a strong and concerned focus on the purpose and practice of everyday research that involves data but is not centrally focused on it as the means and ends of inquiry. Although this 
may seem self-evident and obvious to those social researchers who have been critiquing datafication, evidence-based standards, and big data trends for many years, rearticulation of this stance is an important iteration in the larger nonpositivist spheres of inquiry. Continual reflection on what constitutes data keeps the concept fresh, as something that must be defined rather than taken for granted. It also can allow for reflection on how meaning is being made throughout various moments of the inquiry process.

Data gathering tools are growing more and more refined through sensors and the ubiquity of the Internet of things in our household and personal bodily devices. This is not on the horizon, but a current capacity. Machine learning finds meaningful patterns, which means that big data calculations are more and more accurate. Marketers bid in real time to have the right to show us their advertisement when we use a particular word in a search engine, or land on a particular website. Using calculations from large data aggregators, personal advertising is automatically generated.

In other words, some of the very processes we associate with qualitative research are being automated, whereby machines collect data about individuals, analyze their behaviors, attitudes, and possible future actions, compare this with other people (whose data are also being gathered and analyzed), and use these findings to make decisions about what to show in a search result, an advertisement, or a news feed. The sort of data analytics being conducted now are predicated on the belief somewhere in the chain of stakeholders that the algorithm is working correctly and that the data is representative or unquestionable. The criticism that the these are flawed assumptions may seem banal when the calculation in question is whether or not Netflix recommendation algorithm got it right, but the significance is much more troubling when we consider how these analytics guide predictive policing practices in cities like Los Angeles (Brayne, 2017).

We can't stop machine learning, nor should we want to, but we can question both the source of the data and the simplification of human experience that inevitably accompanies datafication and data analytics (Grinter, 2013; Markham, 2013).

This is an opportunity for qualitative researcher to engage in a critical pedagogy of data literacy simply by talking about how data get defined as objects, how the idea of data fits (or not) into different logics of sensemaking, and how one's framework for defining and conceptualizing data matters (Markham, 2017). My participants and students remind me time and again that they are aware of being tracked, watched, surveilled, and sold to. They knowingly carry on personal and intimate activities in public digital spaces. For the most part, they take this for granted, accepting it as the price of free access and convenience of targeted advertisements, better prediction about their likes and dislikes, and faster directions for getting somewhere or locating and connecting with friends. They, like most youth, tend to define privacy differently than older generations, enacting novel techniques to increase their privacy or at least their sense of privacy (boyd, 2014; Marwick \& boyd, 2012). Increasingly, they enact techniques to control who they share information with and are very careful about which pictures they post to which platform. To make these decisions, they use a complex set of criteria related to their understanding of privacy, publicity, friendships, self-branding, and surveillance.

Still, although they know many strategies for working around tracking and data protection concerns, they-like most of us - have impoverished knowledge about how data are being operationalized and with what consequences. Most have some idea that there are larger implications but are unclear about these beyond their personal sphere. So rather than try to convince them that big data are bad or wrong or that all big data metrics yield only a partial representation of a limited sample (Baym, 2013), I encourage them to learn for themselyes that they are being tracked and calculated as data in ways they cannot see or don't notice. There is a side benefit to this: they begin to pay attention to how this might serve others' interests more than their own. Then, they can begin to see the flaw in the idea that we simply trade priyacy for convenient access.

\section{Nurture the Value and Relevance of Nonquantifiable (Qualitative) Ways of Knowing}

What is qualitative research in an era where data are being defined as the basic unit of what we study? As the concept of data - big or small — grows more dominant, how does it get defined? Pressuring ourselves as researchers to address these questions in our own studies can help describe the qualities of the nonquantitative position, which involves at its core a particular orientation toward the subject of study that differs from other stances.

In such times, I believe it is imperative for scholars who use methods traditionally associated with more qualitative traditions to shift away from identity markers like "qualitative" and "quantitative" toward more refined descriptions of what they/we do in the process of inquiry. This is not just a rhetorical decision to situate between two paradigms, but a matter of epistemological clarity. Defining and delimiting our relationship to and with the material we work with in the course of a study from start to finish is not just a matter of resisting data-oriented mind-sets. It is a proactive effort to build critical data literacy skills for the public, who, by understanding more qualitative orientations, push back against popular and simplistic definitions of what data are as well as simplistic formulas about how computation works.

I find myself talking about qualitative research all the time when I teach or give workshops and talks about methods, but 
I find myself using the term "qualitative" less and less, because it glosses the epistemological. The key to building reflexive interpretive logics is to be inductive in the pedagogical approach and to treat all inquiry as the same set of processes. Starting from this basic and somewhat simplistic premise, we can explore what logics and practices we tend to use to make sense of situations. We can then reverse engineer our habitual ways of knowing to find what methodological camp we might fit into.

Without the baggage of disciplinary research methods terminology, participants tend to use what seasoned methods teachers would call reflexive interpretive methods to explore their own uniqueness, relationality, and the complexity of human decision making. They easily discuss how they know a friend is a real friend, a Facebook friend, or a Frenemy, or what makes relationships happen, and then they can be taught to examine what evidence they're using to reach these analytical conclusions. They can deconstruct the idea of the "quantified self" and articulate how certain data are useful and other data would be impossible to gather. They raise questions about whether big data or data science can be truly objective and while my qualitative sensibilities cringe that they would even ask such questions, I let them carry this discussion through to the end, where they, like many of us, reach the same conclusion I would have started with, only they own the knowledge in ways my telling could never allow.

When I do this as part of a class, the more theoretical definitions come only at the end, when we decide we should put a label on the experiments, reflections, and the approaches toward studying their lived experience. Then, we begin to use terms like phenomenology, discourse analysis, grounded theory, ethnography, or critical theory. We start to borrow from relevant scholars to add depth and larger insights to their explanations and help them make arguments that are accepted as currency within the academic institution. The questions of "so what?" or "who cares?" broaden out from the focus on the self and inevitably, we start to recognize and address larger matters about how our worlds are in the making-something we could label "matters of concern", "matters of care" (Barad, 2007), or much earlier, "worlding" (Peirce, 1958).

The point of this effort is to allow qualitative research sensibilities to seep into everyday sensemaking. This seems a ridiculous statement, since it accurately describes what everyday sensemaking actually is. Still, we find ourselves in an epoch where it has become (again) necessary to promote a nonquantitative approach as a counterbalance to the rapid turns toward quantification, datafication, and computation. Citizens have become encouraged to become citizen scientists who use quantitative logics and methods to make sense of their physical exertions, diet, relationships, and reputation through data-driven metrics and statistical analysis. Many social scientists now use the label data scientists. There is, in short, a widespread assumption that quantitative logics are the core of human experience and assessment, which we can critique as incomplete, but cannot counter. Machine learning only grows more accurate in its predictive abilities, which proves the quantitative logic over and over. Qualitative approaches provide the only lens to explore uniqueness, anomalies that cannot be explained numerically, and, most importantly, to interpret, or give meaning to, the amazing patterns that can be found through (big) data analytics.

\section{Conclusion}

This article is really more of a manifesto. It suggests that we should stop simply rejecting the concept of data. Instead, we (academics, teachers, scholars) should use our long training in pedagogy and teaching and our knowledge of interpretive and inductive/emergent methods of analysis to create better literacies about what data can mean. We can help people find modes and means of critically examining and understanding the contexts within which they are drawn into a neoliberal position through the seemingly innocuous practices of such things as making and sharing images, clicking on links, turning on the smartphone's GPS.

Continual monitoring, quantification, datafication, and computation is, in other words, a present reality we have not yet figured out how to resist without being disconnected completely. Some people try by, for example, disconnecting from social media, but this is becoming less possible for digitally saturated cultures, where government services are increasingly platform-ized and where everyday communication is difficult if not impossible without smart devices. Others use more or less tech-savvy means of circumventing, gaming, or confusing the platform- or service-level algorithmic logics, to avoid being categorized and co-opted as an ideal consumer for specially targeted products or being pigeonholed into a narrowband neoliberal subject position. This is ever more difficult as machine learning grows more sophisticated and multiple miniscule actions are combined across devices and contexts.

By tugging on one or two threads of this tangle, we can trouble (and help others trouble) what a particular part of someone's life might mean, in terms of privileging certain people and marginalizing others, commoditizing someone's creativity without pay, unknowingly narrowing and biasing one's view of news, tracking one's activities for no valid or valuable reason, or collecting data because it might be used in the future, without any exchange value, and so on.

Once we give people the tools to analyze one or two threads, they can find and examine other threads for themselves later, because they have discovered and trained within themselves the capacity to ask specific kinds of questions. They can help their friends, parents, and children do the same. This is how the agency part of the structuration process works, if we follow Giddens (1984) notion of the dynamic relation of structure and agency. Or, using Gramsci, it can also be seen as a process of organic ideology, whereby people within a hegemonic system become aware of the 
deep structures (Mumby, 1988) and act as social agents to build alternate ideologies (Ramos, 1982). Regardless of which conceptual history we use to explain this process, this is how critical data and digital literacy works. Through a process of first intellectualizing and then enacting other possibilities, people can become more capable of making more conscious choices. With a stronger intellectual framework undergirding their analyses, they can resist in ways that do not oversimplify the way that the sociotechnical economies of the digital age work. Academics have a lot to add to these conversations when we turn our attention outward.

\section{Declaration of Conflicting Interests}

The author(s) declared no potential conflicts of interest with respect to the research, authorship, and/or publication of this article.

\section{Funding}

The author(s) received no financial support for the research, authorship, and/or publication of this article.

\section{Note}

1. My fellow grad student colleagues at Purdue deserve a shoutout for this technique, particularly Rachel Pekora, who if my memory serves correctly, created the condensed version of the story.

\section{References}

Bødker, S. (2003). A for alternatives. Scandinavian Journal of Information Systems, 15, 87-89.

Barad, K. (2007). Meeting the Universe Halfway: Quantum Physics and the Entanglement of Matter and Meaning. Durham, NC: Duke University Press.

Baym, N. (2013). Data not seen: The uses and shortcomings of social media metrics. First Monday, 18(10). doi:10.5210/ fm.v18i10.4873

Boellstorff, T. (2013). Making big data, in theory. First Monday, 18(10). doi:10.5210/fm.v18i10.4869

boyd, d. (2014). It's complicated: The social lives of networked teens. New Haven, CT: Yale University Press.

boyd, d., \& Crawford, K. (2012). Critical questions for big data. Information, Communication \& Society, 15, 662-679.

Brayne, S. (2017). Big data surveillance: The case of policing. American Sociological Review, 82, 977-1008.

Brock, A. (2015). Deeper data: A response to boyd and Crawford. Media, Culture, \& Society, 37(7), 1-5.

Clarke, A. (2003). Situational analyses: Grounded theory mapping after the postmodern turn. Symbolic Interaction, 26, 553-576.

Freire, P. (1968). Pedagogy of the oppressed. New York, NY: Herder \& Herder.

Garfinkel, H. (1967). Studies in ethnomethodology. Englewood Cliffs, NJ: Prentice Hall.

Giddens, A. (1984). The constitution of society. Berkeley: University of California Press.

Gitelman, L. (2013). Raw data' is an oxymoron. Cambridge, MA: MIT Press.
Gramsci, A. (1971). Selections from the prison notebooks. New York, NY: International Publishers. (Original work published 1937)

Grinter, B. (2013). A big data confession. Interactions, 20(4), 10-11.

Hesse-Biber, S., \& Leavy, P. (2010). Handbook of emergent methods. New York, NY: Guilford Press.

Latour, B. (2004). Why has critique run out of steam? From matters of fact to matters of concern. Critical Inquiry, 30, 225-248.

Markham, A. (2013). Undermining "data": A critical examination of a core term in scientific inquiry. First Monday, 18(10). doi: $10.5210 / \mathrm{fm} . v 18 \mathrm{i} 10.4868$

Markham, A. (2017). Troubling the concept of data in digital qualitative research. In U. Flick (Ed.), Handbook of qualitative data collection (pp. 511-523). London, England: SAGE.

Markham, A., \& Dougherty, M. (2014). Social Distortion. What we see through the artifacts of digital living? Aarhus, Denmark: Aarhus University.

Marwick, A., \& boyd, d. (2014). Networked privacy: How teenagers negotiate context in social media. New Media \& Society, 16, 1051-1067.

Mumby, D. (1988). Communication and power in organizations. Norwood, NJ: Ablex Press.

Peirce, C. S. (1958). The principles of phenomenology: The categories in detail. In C. Hartshorne \& P. Weiss (Eds.), Collected papers of Charles Sanders Peirce (Vol. 1, pp. 148-180) Cambridge, MA: Harvard University Press.

Ramos, V. (1982). The concepts of ideology, hegemony, and organic intellectuals in Gramsci's Marxism. Theoretical Review, 30, 3-8.

Tiidenberg, K., Markham, A. N., Pereira, G., Rehder, M., Sommer, J., Dremljuga, R., \& Dougherty, M. (2017). "I'm an addict" and other sensemaking devices: A discourse analysis of selfreflections of lived experience on social media. Proceedings of the 8th International Conference on Social Media \& Society, Article No. 21. Retrieved from http://dl.acm.org/cita tion.cfm?doid $=3097286.3097307$

\section{Author Biography}

Annette N. Markham ( $\mathrm{PhD}$, Purdue University, 1997) is a professor of Information Studies and Digital Design at Aarhus University in Denmark and affiliate professor of Digital Ethics at Loyola University in Chicago. One of the world's pioneering researchers of digital culture, she has for more than 20 years studied the macro- and micro-level social impacts of social media, digital interfaces, and datafication. She is well-known for her interpretive ethnographic approaches to digital identity, relationships, and cultural formations. as well as for her innovations in ethical practices for studying digitally saturated social contexts. Her early work is well-represented in her first book Life Online (Alta Mira, 1998) and her edited collection on methods for studying digital culture (Internet Inquiry, Sage, 2009, with Nancy Baym). Her more recent work focuses on conceptual frameworks and metaphors that enable ethical and nuanced approaches to the study of culture in a digital era, and can be found in a variety of international journals and edited books (For more information and links visit http://annettemarkham.com). 